\title{
Atypical findings in the left ventricular outflow tract in a patient with hypertrophic cardiomyopathy
}

\author{
Anna Fojt, Michał Marchel, Adam Rdzanek, \\ Janusz Kochanowski, Grzegorz Opolski
}

Department of Cardiology, Medical University of Warsaw, Poland

This study describes the case of a female patient with left ventricular hypertrophy $(\mathrm{LVH})$ who was admitted to the hospital due to the heart failure symptoms exacerbation.

In the transthoracic echocardiography (TTE) an abnormal flow to the left ventricular outflow tract (LVOT) was observed (Fig. 1). No definite source of this phenomenon was identified, however, Doppler examination demonstrated a continuous jet, with a predominant diastolic component, consistent with coronary artery flow. The subsequent transesophageal echocardiography revealed two small coronary artery fistulas communicating with the left ventricle.

Selective coronary angiography showed normal epicardial coronary arteries without significant stenoses. The examination also confirmed the presence of two coronary artery fistulas, originating from the large septal branch of the left anterior descending artery and from the right coronary artery. Both fistulas seemed to communicate, in close proximity to each other, with the left ventricle, in the area corresponding to the TTE, abnormal LVOT flow was observed.

The etiology of coronary fistulas may be congenital or traumatic. They involve abnormal termination of a coronary artery, usually the right coronary, into a cardiac chamber or other vascular structures, such as vena cava, the pulmonary artery, or pulmonary veins.

In the present case, rarely seen fistulas originating from different coronary arteries and communicating with the same area of the left ventricle were most likely of congenital origin. Taking into account the dubious relationship between observed vessel anomaly, LVH and the heart failure symptoms, no specific intervention was recommended.

Conflict of interest: None delcared

Address for correspondence: Dr. Michał Marchel, Department of Cardiology, Medical University of Warsaw, ul. Banacha 1a, 02-097 Warszawa, Poland, tel: +48 5001187219, fax: +48 22599 19 57, e-mail: michal.marchel@wum.edu.pl 


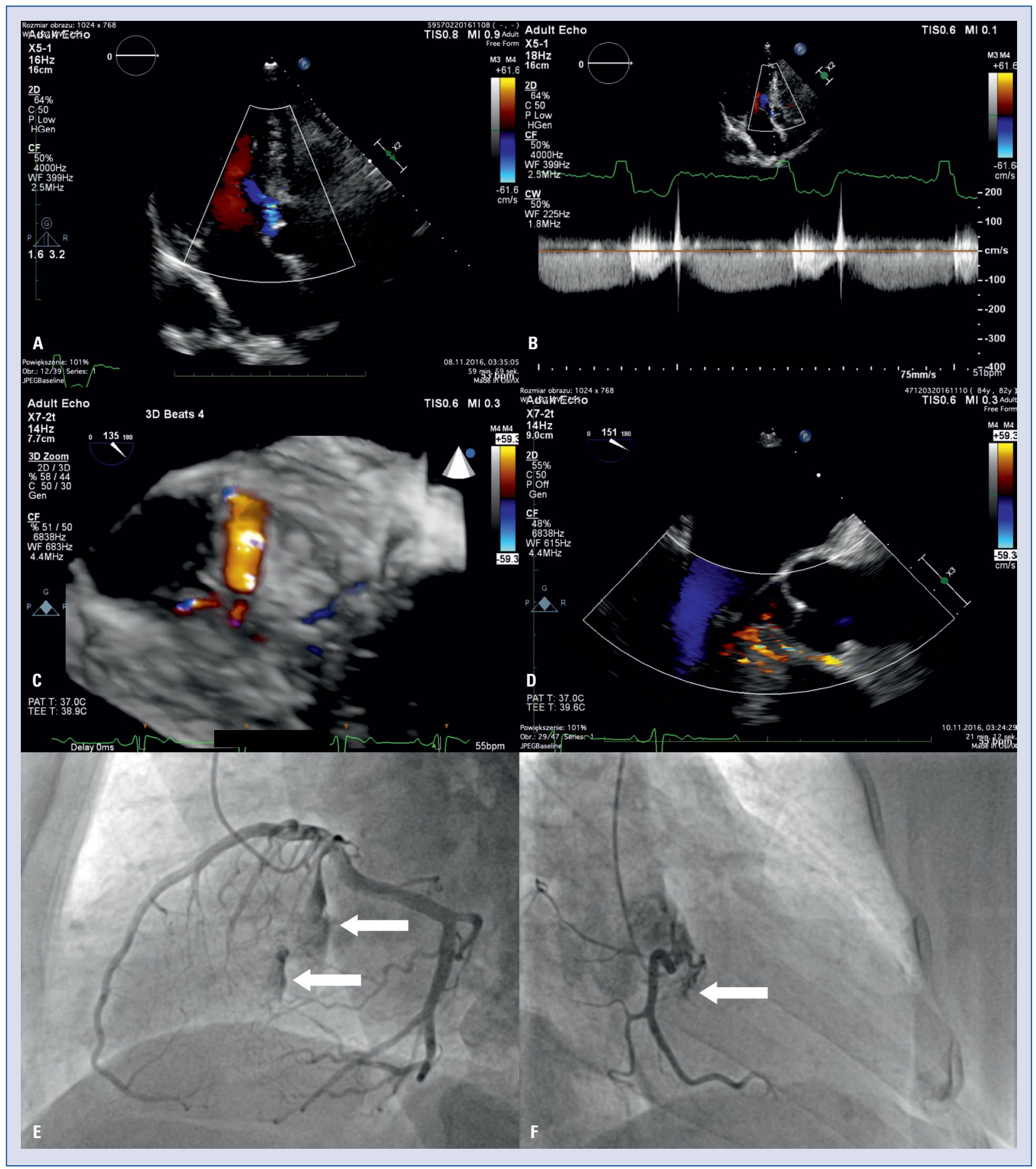

Figure 1. A, B. Transthoracic echocardiography; C, D. Transesophageal echocardiography; E, F. Selective coronary angiography. Abnormal flow in the left ventricular outflow tract (LVOT) in three chamber view (A) with a predominant diastolic component in Doppler examination (B). Both fistulas communicate, in close proximity to each other, with the LVOT (C, D). Selective angiography of coronary arteries (E - left, F — right) show two coronary artery fistulas, originating from the large septal branch of the left anterior descending artery and from the right coronary artery. 